\title{
Jovian electron modulations by the solar wind interaction with the magnetosphere
}

\author{
F. Tsuchiya, A. Morioka, and H. Misawa
}

\author{
Planetary Plasma and Atmospheric Research Center, Graduate school of Science, Tohoku University, Sendai, 980-8578, Japan
}

(Received August 5, 1997; Revised June 25, 1999; Accepted August 27, 1999)

\begin{abstract}
Variations of energetic electrons released from the Jovian magnetosphere are investigated in detail by using data from three spacecraft, Pioneer 10, 11, and Ulysses. Analyses with the three spacecraft data confirmed that the Jovian electrons are modulated by the dynamic pressure of the solar wind at the position of Jupiter. It is also found that the release rate of energetic electrons is controlled by the polarity of IMF having dawn-dusk or latitudinal dependence. The enhancements of released electron flux were detected during the toward polarity of IMF when the spacecraft were located in the upstream or dawn side of Jupiter. On the other hand, Ulysses detected enhancement in away polarity of IMF when the spacecraft was on the duskside of southern hemisphere. We made a simulation to reproduce the electron variation of Jovian electrons observed by Pioneer 11 considering the convective and diffusive transport in the interplanetary medium. According to the simulation, the observed enhancements of electron flux originating from Jupiter are well represented by the coupling of both the source modulation at the Jovian magnetosphere and the transport effect in the interplanetary medium.
\end{abstract}

\section{Introduction}

In 1973, when Pioneer 10 was approaching Jupiter, unexpected relativistic electron increases were detected in interplanetary space (Chenette et al., 1974; Simpson et al., 1974; Teegarden et al., 1974). The intensity of electron fluxes increased as Pioneer 10 approached Jupiter, showing that the relativistic electrons accelerated in the Jovian magnetosphere were released into the interplanetary medium. According to the Pioneer 10 observation, a large number of energetic electrons were found not only in the radiation belt formed around the inner magnetosphere but also throughout the middle and outer magnetospheres even just inside the magnetopause. It shows clear contrast with those in Earth's magnetosphere.

The electron fluxes observed in interplanetary space showed two types of variations, that is, 10-hour modulation associated with the Jovian rotation (Chenette et al., 1974; Schardt et al., 1983; Ferrando et al., 1993b; Simpson et al., 1993) and large amplitude variations with the period of the solar rotation ( $~ 25$ days) (Conlon and Simpson, 1977; Conlon, 1978; Chenette, 1980). Two processes are considered as the cause of the latter type of flux variation; (1) modulation of transport in the interplanetary medium and (2) modulation of release from the Jovian magnetosphere.

Previous studies claimed that large amplitude variation of Jovian electron flux observed in interplanetary space were explained by "barrier" effect of corotating interaction regions (CIRs) in the solar wind (Conlon and Simpson, 1977; Conlon, 1978; Chenette, 1980). In contrast to the modulation in interplanetary space, Chenette (1980) first suggested the possibility of modulation of release rate from the Jovian magnetosphere. Morioka and Tsuchiya (1996) showed that

Copy right $(\mathcal{C})$ The Society of Geomagnetism and Earth, Planetary and Space Sciences (SGEPSS); The Seismological Society of Japan; The Volcanological Society of Japan; The Geodetic Society of Japan; The Japanese Society for Planetary Sciences. the depression (enhancement) of the dynamic pressure of the solar wind at Jupiter causes the increase (decrease) of the release rates of Jovian electrons from the magnetosphere, by using cross-correlation analyses. They also made the simplified model considering both the source effect at Jupiter and the barrier effect by CIRs to reproduce the flux variation of Jovian electrons detected in interplanetary space. Chenette (1980) proposed that an additional modulation might be caused by the polarity of the interplanetary magnetic field (IMF). He found, using energetic electron data observed by IMP 8 in 1974, that more electrons were released from the Jovian magnetosphere in the toward sector of IMF than in the away sector. Similar trend was found in the Pioneer 11 data obtained during the same period. Morioka et al. (1997) showed significant relationship between the IMF polarity at Jupiter and the Jovian electrons in interplanetary space by using the Pioneer 11 data.

Transport of Jovian electrons in the interplanetary medium is described by a convective diffusion model (Chenette et al., 1977; Conlon, 1978; Hamilton and Simpson, 1979; Ferrando et al., 1993a,b; Simpson et al., 1993). Conlon (1978) and Chenette (1980) applied a time dependent solution of a convective diffusion equation to the transport of Jovian electrons. Conlon (1978) calculated the convective diffusion equation and accounted for the increase of Jovian electrons observed by Pioneer 11, assuming that Jovian electrons are emitted constantly from the magnetosphere and begin to be transported after passage of a CIR at Jupiter.

In this paper, we first confirm the previous results of Morioka and Tsuchiya (1996) by using the data from three spacecraft; i.e., Pioneer 10, 11 and Ulysses, and further show detailed relationship between Jovian electron fluxes and the polarity of the IMF. We then introduce a time variable source into the convective diffusion model to examine the effect of 
Table 1. Data sets from Pioneer 10, 11 and Ulysses.

\begin{tabular}{lll}
\hline Spacecraft and data & Instrument & Reference \\
\hline $\begin{array}{l}\text { Pioneer 10, 11 } \\
\text { High energy electron }\end{array}$ & Charged Particle Instrument & \\
$\begin{array}{l}\text { Solar wind plasma } \\
\text { Magnetic field (IMF) }\end{array}$ & Plasma Analyzer Experiment & Chenette et al., 1974 \\
\hline Ulysses & & Wolfe et al., 1974 \\
High energy electron & Cosmic Ray and Solar Particle Investigations & Simpson et al., 1992 \\
Solar wind plasma & Solar Wind Obs. Over the Poles of the Sun & Bame et al., 1992 \\
Magnetic field (IMF) & Helium Vector Magnetometer & Balogh et al., 1992 \\
\hline
\end{tabular}

diffusive electron transport in the interplanetary medium.

\section{Data and Spacecraft Trajectories}

The data used in this paper were obtained from the Coordinated Heliospheric Observations (COHO) database (Cooper et al., 1991) provided by the National Space Science Data Center (NSSDC). High-energy electron data and the solar wind parameters are provided from the three spacecraft, i.e.; Pioneer 10, 11, and Ulysses. Table 1 shows the list of the data sets. In this paper, two- or three-year period of data for each spacecraft including the encounter with Jupiter was selected for the analysis.

We used data of high energy electron counting rates in a 7-17 MeV energy range measured from the Pioneer 10 and 11 spacecraft (Chenette et al., 1974). The time resolution of the provided data is 1-hour. As for the Ulysses spacecraft, we used 7-170 MeV electron counting rates (10-min. resolution) measured with the Kiel Electron Telescope instrument (KET; Simpson et al. (1992)). In this study, enhanced electron fluxes above the background level were treated as of Jovian origin (Chenette et al., 1974; Teegarden et al., 1974) except for some solar flare events which were identified by referring proton counting rates and were removed from the present analyses. Short data gaps were supplemented with a firstorder interpolation.

The solar wind plasma data are provided with 10-minute averaged value for Pioneers (Wolfe et al., 1974) and several minutes value for Ulysses (Bame et al., 1992), and the IMF data are also provided with 10-minute averaged value for Pioneers (Smith et al., 1974) and 1-minute value for Ulysses (Balogh et al., 1992). The dynamic pressure of the solar wind is calculated from the density and the velocity data. The IMF data are represented in RTN coordinate system, where the $R$ axis is the radial direction from the sun to a spacecraft, the $T$ axis is the cross product of the solar rotation axis and the $R$ axis, and the $N$ axis is the cross product of $R$ and $T$. When a spacecraft is located near the solar equatorial plane, $T$ and $N$ axes indicate the south-to-north and dusk-to-dawn directions, respectively.

Figure 1 shows the trajectories of the spacecraft in the coordinate system where the Sun-Jupiter line is fixed. Panels (a) and (b) in the figure show the projections onto the Jovian orbital plane and onto the meridian plane of the sun, respec-
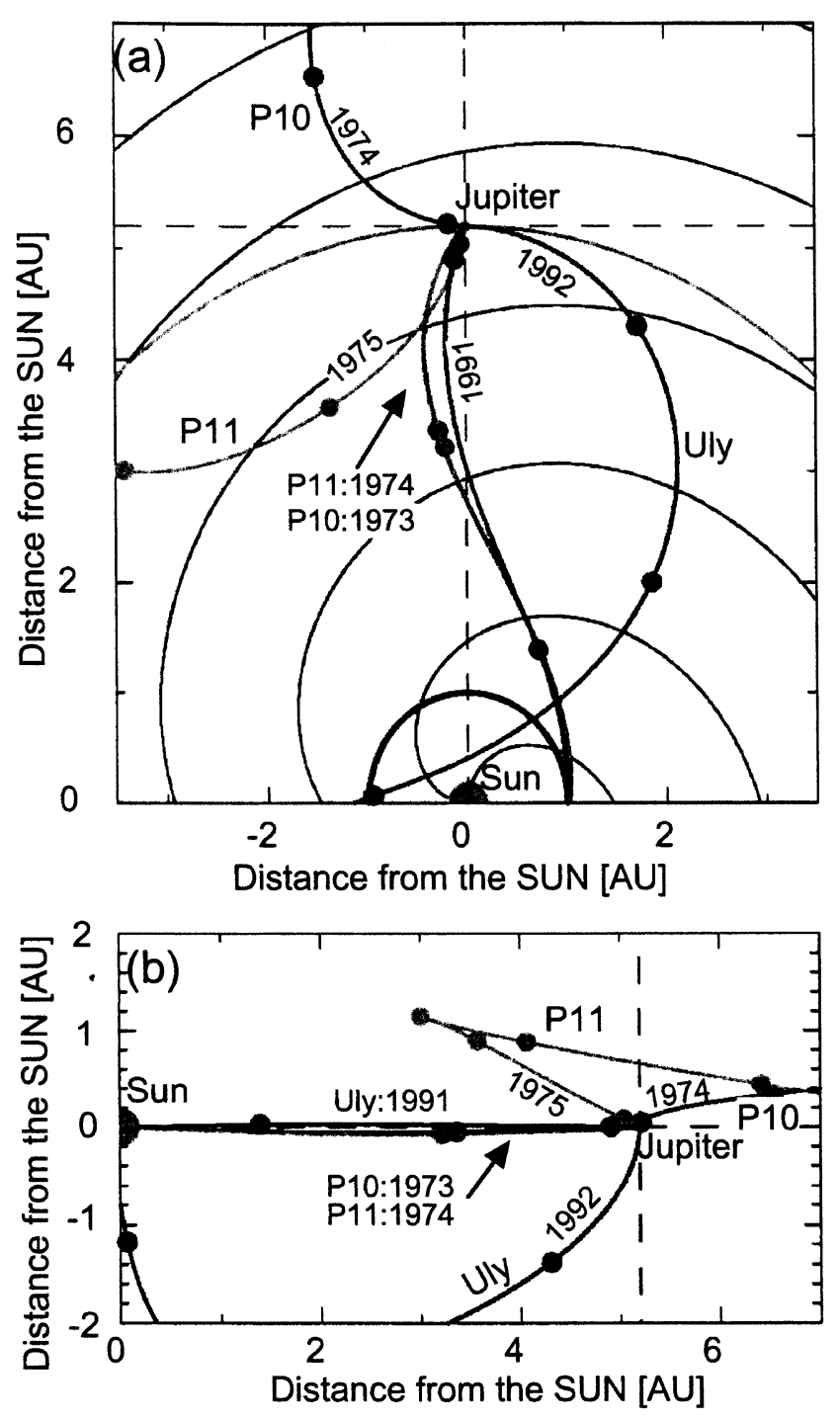

Fig. 1. Trajectories of three spacecraft. (a): projection onto the Jovian orbital plane, (b): projection onto the meridian plane of the sun.

tively. Inbound trajectories of the three spacecraft lay close to the solar equatorial plane and have nearly the same heliospheric longitude as Jupiter when spacecraft were within $2 \mathrm{AU}$ from the planet, that is, both the maximum longitudi- 

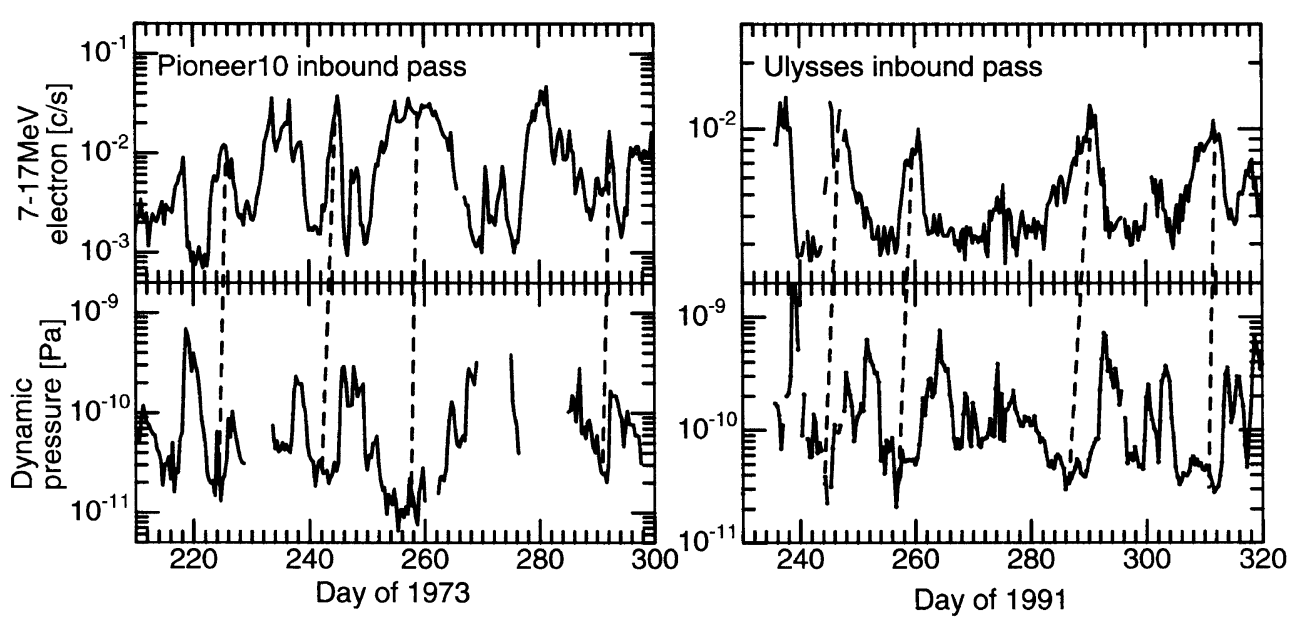

Fig. 2. 8-hour averaged plot of high energy electron counting rates and the in-situ solar wind dynamic pressure measured by Pioneer 10 (left) and Ulysses (right) during their inbound passes.

nal and latitudinal separations between spacecraft and Jupiter were less than several degrees. Therefore, the solar wind that passed the spacecraft also passed by Jupiter. In the outbound pass, Pioneer 10 and 11 traveled dawn side of Jupiter, and the Ulysses spacecraft traveled high latitude dusk side in southern hemisphere of Jupiter. These various trajectories made it possible to investigate local time dependence of high-energy Jovian electrons.

\section{Analyses}

\subsection{Jovian electron modulation by the solar wind vari-} ation

Morioka and Tsuchiya (1996) found the anti-correlation between the Jovian electron flux and the solar wind dynamic pressure in the data of the Pioneer 11's inbound pass. First, we re-examine the anti-correlation by using observations from the inbound passes of Pioneer 10 and Ulysses, and the outbound pass of Pioneer 11. Figure 2 shows examples of solar wind dynamic pressure and high-energy electron counting rates observed by Pioneer 10 and Ulysses. In both cases, anti-relations between electron counting rates and the dynamic pressure are observed with some time delay. The sense of the delay is the same as that was found in the Pioneer 11 data by Morioka and Tsuchiya (1996). To confirm the result of Morioka and Tsuchiya (1996), the relations between Jovian electrons and the solar wind were examined by crosscorrelation analysis. Cross-correlation coefficients were calculated for every 50-day data set of 8-hour averaged value, and the 50-day window was scanned with 1-day step through the year of interest.

Figure 3 shows the dynamic correlation coefficients calculated from the Pioneer 11's inbound (a) and outbound (b) passes, the Pioneer 10's inbound pass (c), and the Ulysses' inbound pass (d). All spacecraft were located in the upstream region of the Jovian magnetosphere during these periods. The positive lag time indicates that the solar wind leads Jovian electrons. The correlation coefficients are displayed in color code. Solid lines superposed on the diagram indicate the solar wind travel time from the spacecraft to Jupiter calculated from the in-situ solar wind velocity data.
Significant negative correlation (red in color) is recognized in Figs. 3(a) (d). The lag times vary depending on the distance between the spacecraft and Jupiter, consistently with the solar wind travel time.

From these results, we confirmed that the release of Jovian electrons is strongly controlled by the solar wind variations at Jupiter. It is also suggested that this effect is common nature in both solar minimum and maximum periods.

\subsection{Flux variation by the polarity of IMF}

Figure 4 shows peak intensities of electron fluxes observed by Pioneer 11 with respect to the daily averaged $B_{T}$ components of IMF which were time-shifted to the value at the position of Jovian magnetosphere. The period of the data plotted in the figure was selected from day 60 to 330 in 1974 during which electron increases of Jovian origin were clearly identified. The electron intensities are corrected for the $(1 / R)$ effect from the planet, where $R$ is the distance between Pioneer 11 and Jupiter. According to the figure, Pioneer 11 observed the enhanced electron flux during the period of positive $B_{T}$. This result suggests that the polarity of IMF at the position of Jupiter controls the release rate of Jovian electrons.

In Figs. 5(a) (c), the data of the three spacecraft were processed to produce "recurrent diagrams" where the time profile of electron flux was divided by every 26 days (recurrent period of the corotating solar wind stream) and arranged in order. Electron intensity was indicated by color code. Blanks indicate the periods when the spacecraft were in the Jovian magnetosphere, and gray colors indicate lacks of data. The right side of Fig. 5 is the recurrent diagram of the polarity of IMF $\mathrm{B}_{T}$ at Jupiter. The toward (away) polarity of the IMF is indicated by red (blue) color. Figure 5(a) is the recurrent diagrams of the Pioneer 11 observation during 1974-1975. The spacecraft was traveling upstream region of Jupiter before the encounter with the planet (Dec., 1974). After the encounter, the spacecraft was in the dawn side of upstream region. It is clear that electron enhancements occurred preferentially in the toward magnetic field sector. Figure 5(b) shows that the same feature was found in the Pioneer 10 trajectory in the dawn side of the downstream region of the Jovian magnetosphere. The IMF dependence was not obvious in early 1974 

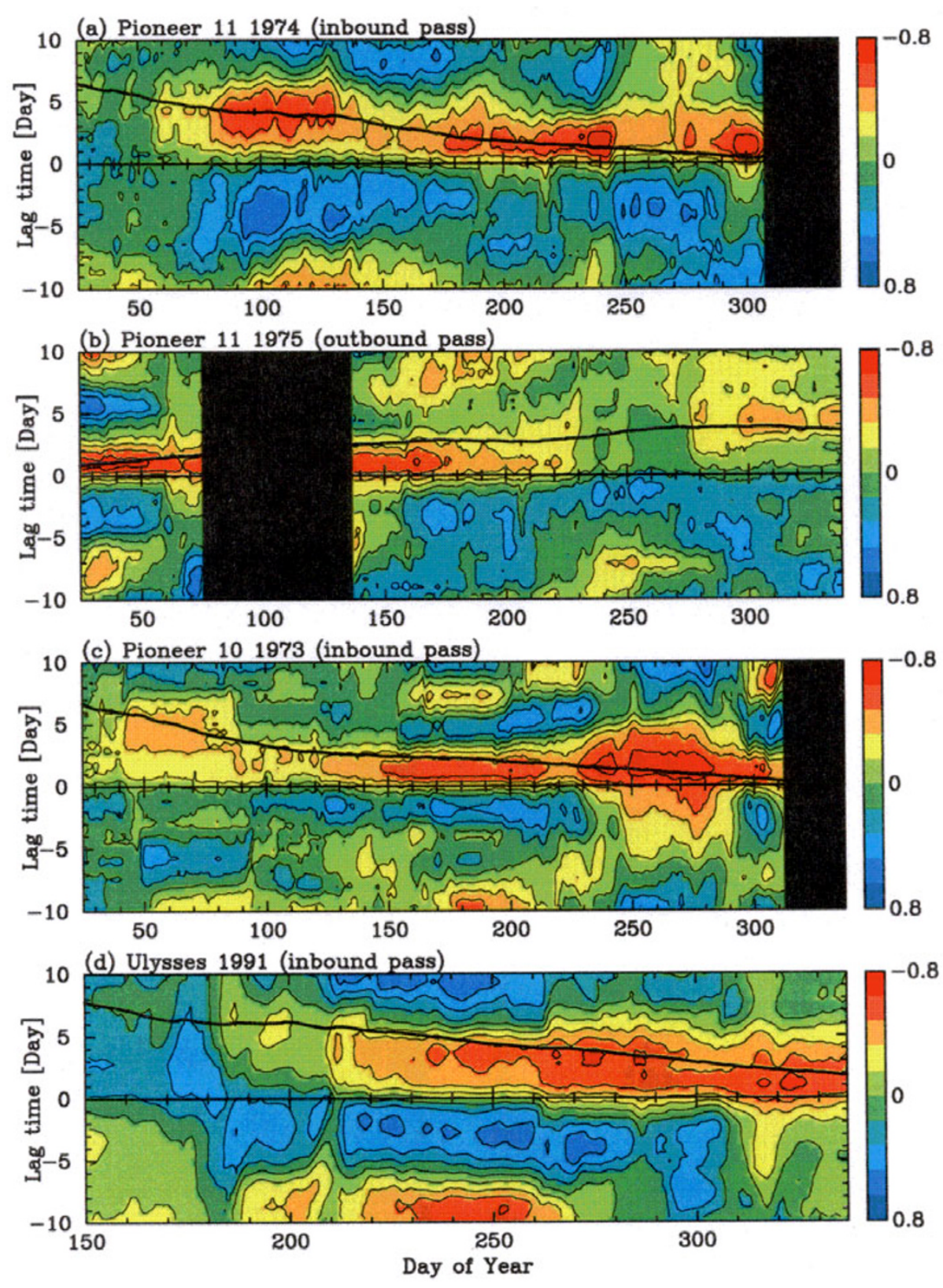

Fig. 3. Dynamic cross-correlations between in-situ dynamic pressure of the solar wind and the 7-17 MeV electron counting rates. Each panel indicates the case of the Pioneer 11's inbound pass (a) (reproduced from Fig. 2 in Morioka and Tsuchiya (1996)) and outbound pass (b), the Pioneer 10's inbound pass (c) and the Ulysses' inbound pass (d). The enhanced negative coefficients indicated by reddish color code mean the anti-correlation. The positive lag time of cross-correlation coefficients indicates that the dynamic pressure of the solar wind leads the electron rates. The trend of the negative peaks of the cross-correlation coefficients is consistent with the estimated travel time of the solar wind from the spacecraft to Jupiter (solid line).

because the electron detector onboard Pioneer 10 reduced its efficiency over a period of 3-6 months after the passage through the Jovian radiation belts (Pyle and Simpson, 1977). In late 1975, when Pioneer 10 was far from Jupiter, the intensity of Jovian electron fluxes became too small to obtain good $\mathrm{S} / \mathrm{N}$ with respect to background level. It is concluded that the Jovian electron fluxes observed in both the upstream and dawn side regions of Jupiter increase during the period when Jovian magnetosphere was in the toward sector of the IMF.

Figure 5(c) shows the case of Ulysses. The Ulysses spacecraft traveled upstream region of Jupiter in the inbound pass, and dusk-side and high-latitude region of southern hemi- sphere in the outbound pass. In the inbound pass, the electron intensity was enhanced during the period when Jupiter was in the toward polarity sector. It is consistent with the results from Pioneer 10 and 11 . On the other hand, electron enhancements occurred in the away polarity sector in the outbound pass. This means that the control of the IMF polarity on the electron release was switched depending on the Ulysses location with respect to the Jovian magnetosphere.

Table 2 shows a summary of the observed IMF control of Jovian electrons with respect to the position of spacecraft. In the upstream and dawn side of the Jovian magnetosphere, Jovian electron intensities were enhanced when the IMF polarity at Jupiter was toward. Only when Ulysses was lo- 
Table 2. The spacecraft position and the polarity of IMF during the Jovian electron enhancement.

\begin{tabular}{cccc}
\hline Spacecraft & Pass & Position of S/C & Polarity of $\mathrm{B}_{T}$ \\
\hline Pioneer 11 & inbound pass & Upstream & Toward \\
Pioneer 11 & outbound pass & Dawn side of Upstream & Toward \\
Pioneer 10 & outbound pass & Dawn side of Downstream & Toward \\
Ulysses & inbound pass & Upstream & Toward \\
Ulysses & outbound pass & Dusk side of Southern hemisphere & Away \\
\hline
\end{tabular}

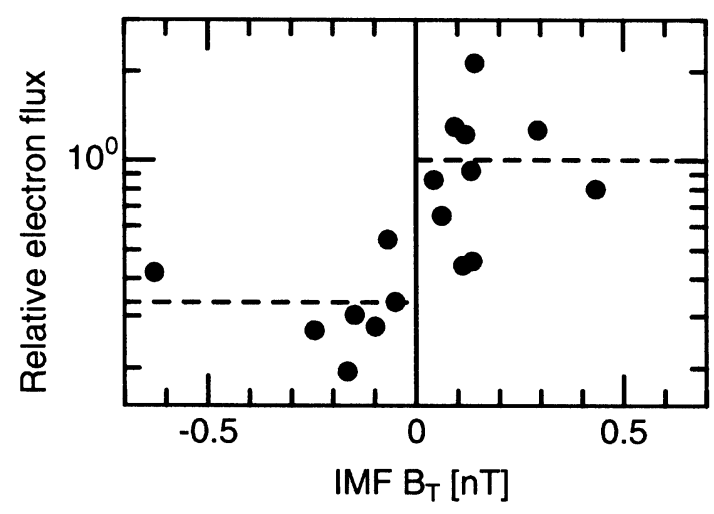

Fig. 4. Peak intensities of Jovian electrons measured by Pioneer 11 with respect to the IMF $\mathrm{B}_{T}$ at Jupiter (shifted by the travel time of the solar wind from the spacecraft to Jupiter). Period of data was selected from day 60 to day 330 in 1974. Intensities of Jovian electrons were corrected for the distance dependence $(1 / \mathrm{R})$. The dashed lines indicate the average of peak intensities during each polarity sector.

cated in dusk side and high latitude region of the southern hemisphere, electron intensities were enhanced in the away polarity sector.

\subsection{Summary of analyses}

From the present analyses, it is proposed that the Jovian electron fluxes observed in interplanetary space are represented by following relation;

$$
f_{\mathrm{obs}} \propto P_{\mathrm{SW}}^{-\alpha} \cdot \beta_{\mathrm{IMF}}
$$

where, $P_{\mathrm{SW}}$ is the dynamic pressure of the solar wind at the planet, and $\alpha$ is a positive parameter that represent the modulation strength by the solar wind. Release efficiency $\beta_{\mathrm{IMF}}$ caused by the IMF polarity is summarized as;

$$
\begin{aligned}
\beta_{\mathrm{IMF}}(\text { toward }) & >\beta_{\mathrm{IMF}} \text { (away) } \\
& \text { at upstream and dawn side regions, } \\
\beta_{\mathrm{IMF}}(\text { toward }) & <\beta_{\mathrm{IMF}} \text { (away) } \\
& \text { at dusk side. }
\end{aligned}
$$

In constant to our analyses, Conlon and Simpson (1977), Conlon (1978), and Chenette (1980) showed that variations of Jovian electron flux were explained by the "barrier" effect of CIRs, which are not taken into consideration in our analyses. It is expected that observed electron variations are mixture of both the barrier and the source effects. As for the source effect, the observed Jovian electron flux responds to the dynamic pressure of the solar wind at the position of Jupiter. Therefore, the observed electron flux decreases (increase) when high (low) dynamic pressure regions of the solar wind interact with the Jovian magnetosphere. On the other hand, in the barrier model, the observed flux level is affected when the CIRs lie between the magnetosphere and a spacecraft because CIRs play a roll of inhibiting the propagation of Jovian electrons. In order to divide source effect from barrier one and evaluate their contributions to the observed electron variations, model calculation should be necessary. We should also consider the transport time of energetic electrons when we examine profiles of the detected electron variation on the basis of the diffusive propagation across the interplanetary field (Conlon, 1978).

In the next section, we calculate the flux of Jovian electrons with the time dependent convective diffusion equation developed by Conlon (1978) considering the source modulation at Jupiter and barrier effect by CIRs independently. The flux calculated is compared with the observed data from Pioneer 11. We also attempt to determine the index $\alpha$ in Eq. (1) more quantitatively.

\section{Diffusive Transport in the Interplanetary Medium}

\subsection{Time-dependent convective diffusion equation}

Conlon (1978) solved the Fokker-Planck equation on assumptions of a point source of electrons at Jupiter, constant diagonal diffusion tensor, and power law spectrum of Jovian electrons. The reduced differential number density $U(t, T, \mathbf{x})$ of particles is expressed as;

$$
U(t, T, \mathbf{x})=\int_{-\infty}^{t} u\left(t^{\prime} \mid t, T, \mathbf{x}\right) d t^{\prime},
$$

where

$$
\begin{aligned}
u\left(t^{\prime} \mid t, T, \mathbf{x}\right)= & C \cdot e^{2 \mathbf{F} \cdot \mathbf{D}} A^{*}\left(t^{\prime}, T\right)\left(t-t^{\prime}\right)^{-3 / 2} \\
& \cdot \exp \left[-\frac{D^{2}}{t-t^{\prime}}-\left(F^{2}+\lambda\right)\left(t-t^{\prime}\right)\right] \\
D_{i}= & x_{i} / 2 K_{i}^{1 / 2} \\
F_{i}= & V_{i} / 2 K_{i}^{1 / 2} \\
\lambda= & \frac{1}{3}\left(\frac{\partial V_{i}}{\partial x_{i}}\right)(\gamma-1),(i=1,2,3) \\
C= & \left(8 \pi^{3 / 2} \prod_{i=1}^{3} K_{i}^{1 / 2}\right)^{-1}
\end{aligned}
$$



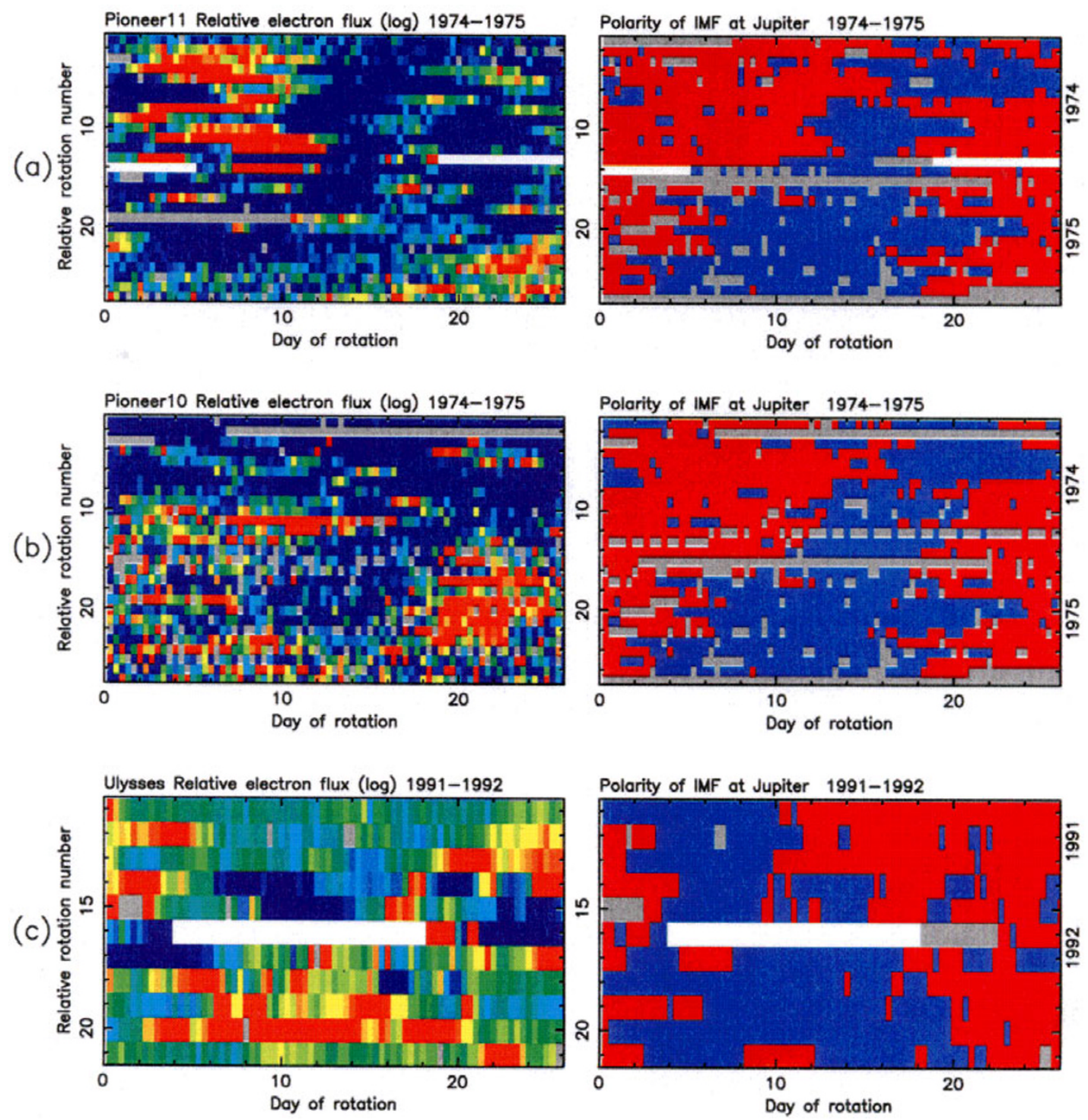

Fig. 5. The recurrent diagrams of 7-17 MeV electrons (left side) and the polarity of IMF $\mathrm{B}_{T}$ at Jupiter (right side) in the cases of Pioneer 11 (a), Pioneer 10 (b), and Ulysses (c). The electron intensity is corrected for the (1/R) effect and indicated by color code. In the right panels, blue color indicates the away polarity and red color indicates toward. In the case of Pioneer 11, most of solar wind velocity data in 1975 were not provided from the data base, therefore the Pioneer 10 data were applied.

Here $u\left(t^{\prime} \mid t, T, \mathbf{x}\right)$ is a fraction of electrons which have been released from the Jovian magnetosphere at time $t^{\prime}$ and pass the spacecraft at location $\mathbf{x}$ at time $t$, and $A^{*}(t, T)$ is the number of electrons released from the Jovian magnetosphere during a short period from $t$ to $t+d t$. The coordinates used here are based on the IMF direction. $x_{1}$ is the distance between a spacecraft and Jupiter measured along the line of IMF, $x_{2}$ and $x_{3}$ are across the IMF parallel and perpendicular to the ecliptic plane, respectively. $T$ is kinetic energy of an electron, $K_{i}$ are diffusion coefficients, $V_{i}$ is the solar wind velocity, and $\gamma$ is spectral index of Jovian electrons. The diffusion coefficients $K_{i}$ have been estimated from the observed spatial distribution of Jovian electrons in the interplanetary medium (Chenette et al., 1977; Conlon, 1978; Hamilton and Simpson, 1979; Ferrando et al., 1993a,b; Simpson et al., 1993). In our calculation, we consider the electron transport within the equatorial plane (i.e., $K_{3}=0$ ), and use the values determined by Chenette et al. (1977); $K_{1}=1.0 \times 10^{21}$ $\mathrm{cm}^{2} \mathrm{~s}^{-1}$ and $K_{2}=5.0 \times 10^{22} \mathrm{~cm}^{2} \mathrm{~s}^{-1}$.

We introduced a time dependent source into the Conlon's solution Eq. (2) instead of a constant source. Based on results of the Pioneers and Ulysses observations (Figs. 2, 3), the source $A^{*}(t, T)$ is set to be represented by a power low of the dynamic pressure of the solar wind at Jupiter with negative power index. Then, the particle source is expressed as

$$
A^{*}(t, T)=A_{0} T^{-\gamma}\left(A_{1}(t)^{-1} \cdot P_{\mathrm{SW}}(t)^{-A_{2}}\right)
$$

where, $A_{0}$ is a constant, $A_{1}$ is a factor of attenuation of particles caused by shocks of CIRs between an observer and the planet (barrier effect) (Conlon, 1978; Chenette, 1980), and $A_{2}$ indicates the degree of modulation of the release rate caused by the dynamic pressure of the solar wind at Jupiter $\left(P_{\mathrm{SW}}\right) . A_{2}$ corresponds index $\alpha$ in Eq. (1). The IMF polarity effect is not included in Eq. (4). Quantitative determination of $\beta_{\mathrm{IMF}}$ in Eq. (1) is feature problem. As the polarity effect influences only the amplitude of the observed fluxes 
rather than the time dependent profile of variations, we can compare the observation and the model results by adjusting model intensity.

The interval $[-\infty, t]$ for the integral in Eq. (2) has to be represented by some finite value in numerical calculation. To set the interval, we calculated the profile of $u\left(t^{\prime} \mid t, T, \mathbf{x}\right)$ as a function of the time from release. We assume a pulsive release of Jovian electrons at time $t^{\prime}=0$ as

$$
A^{*}(t, T)=A_{0} T^{-\gamma} \cdot \delta\left(t^{\prime}\right) .
$$

where, $A_{0}$ is a constant and $\delta(t)$ is the Dirac's $\delta$-function. Substituting Eq. (5) into Eq. (3), we obtain

$$
\begin{aligned}
u\left(t^{\prime}=\right. & 0 \mid t, T, \mathbf{x}) \\
= & A_{0} T^{-\gamma} \cdot C \cdot t^{-\frac{3}{2}} \\
& \cdot \exp \left[2 \mathbf{F} \cdot \mathbf{D}-\frac{D^{2}}{t}-\left(F^{2}+\lambda\right) t\right] .
\end{aligned}
$$

Figure 6 shows the electron flux calculated from Eq. (6) as a function of time $t$ after the pulsive release of Jovian electrons. The solid, dashed, and dotted lines in the figure show profiles of the electron flux at $0.17 \mathrm{AU}, 1.0 \mathrm{AU}$, and 1.5 $\mathrm{AU}$ from Jupiter, respectively. As the distance from Jupiter to the spacecraft increases, the profile of electron flux becomes flat. From Fig. 6, we found that most of electrons are transported at the distance of the spacecraft within 20 days after release. The contribution of "old" electrons which were released more than 20 days before is less than 1/30 of the peak flux just after the electron release. Therefore, we set the interval from $t-20$ days to $t$.

Our calculation of Jovian electron fluxes is summarized as follows;

$$
\begin{aligned}
U(t, T, \mathbf{x}) & \\
= & C \cdot e^{2 \mathbf{F} \cdot \mathbf{D}} \int_{t-20 \text { days }}^{t} A_{0} T^{-\gamma}\left(A_{1}(t)^{-1} \cdot P_{\mathrm{SW}}(t)^{-A_{2}}\right) \\
& \cdot\left(t-t^{\prime}\right)^{-3 / 2} \\
& \cdot \exp \left[-\frac{D^{2}}{t-t^{\prime}}-\left(F^{2}+\lambda\right)\left(t-t^{\prime}\right)\right] d t^{\prime} .
\end{aligned}
$$

Effect of the source modulation, the barrier effect, and coupling both are investigated by using several sets of $A_{1}$ and $A_{2}$ which are listed in Table 3. Source modulation caused by the dynamic pressure of the solar wind is considered in model 1 (source model). According to Morioka and Tsuchiya (1996), the index $A_{2}$ is between 1.0 and 2.0. In the calculation, we assumed to be $A_{2}=2.0$ (we will check this assumption later), while the attenuation factor $A_{1}$ is fixed to be 1.0 (no barrier effect). We also consider barrier (model 2) and coupling (model 3) models. The barrier model is set to be $A_{2}=0$ (no source modulation). In both models, barrier effect is simulated by the attenuation of the source. The attenuation factor $A_{1}$ is set to be 10.0 during the period when a forward or reverse shock of CIR exists between Pioneer 11 and Jupiter, and 1.0 for other periods. Passage of CIRs by Pioneer 11 was identified from the IMF and the solar wind velocity data (Smith and Wolfe, 1976). Periods of CIRs passing Jupiter were estimated from the solar wind travel time from Pioneer 11 to Jupiter. In all models, $A_{0}$ is used to adjust the calculated

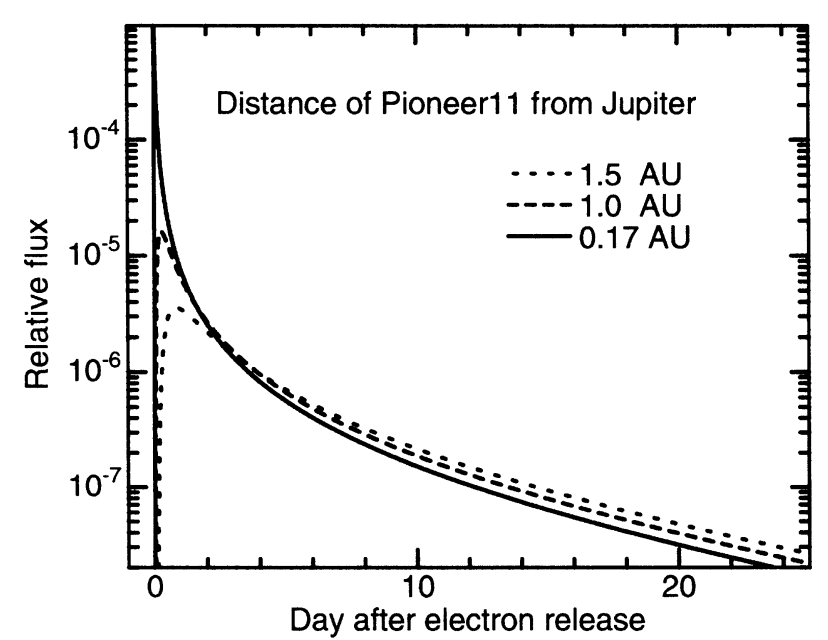

Fig. 6. Decay profiles of relative electron fluxes transported to Pioneer 11 after the pulsive electron flux was released from Jupiter.

value to the observed electron intensity, because our interest is on the time dependent profile of the electron flux.

\subsection{Results of calculation}

The calculation for model 1 where the barrier effect is not taken into account is compared with the observation in Fig. 7. In the figure, thick line indicates the calculated intensity variation of Jovian electrons and thin line indicates 7-17 MeV electron counting rates observed at Pioneer 11 during the period of day 215-325 in 1974 (0.5 0.05 AU from Jupiter). The calculated fluxes were normalized by the observed value at day 285.5 (asterisk in Fig. 7). The source model well reproduces the variation observed by Pioneer 11. During the period from day 284-295 when there was no CIR between the spacecraft and Jupiter, the dynamic pressure of the solar wind changed by factor of 2-5 in the time scale of a few days (not shown here). Therefore, the source rate is expected to have small amplitude variation during this period. Variation by the model coincides with the observation very well, which means that source modulation has predominant contribution to the observed electron variation. Around day 240 and 265 , the observed amplitudes are larger than the calculated one. These disagreements may be caused by the effect of the IMF polarity, as we do not take it into account in Eq. (4). For more precise discussion, it is necessary to determine the value of $A_{0}$ which is a function of the IMF polarity.

Figure 8 shows an example to examine the effects of the source modulation and the attenuation by CIRs in detail. Panel (a) in the figure shows electron flux calculated for source model (model 1; thick line) superposed on the observed electron profiles (thin line). The normalization factor is different from that used in Fig. 7 because of the IMF polarity effect described previously. The source model well agrees with the observed variations again. Now we check the value for $A_{2}$ adopted here (2.0) is reasonable or not. We tested another case where $A_{2}$ was set to be 1.0 (here after referred as model 1') because Morioka and Tsuchiya (1996) showed that the index $A_{2}$ ranged between 1.0 and 2.0. Dashed line in the panel (a) is the result of model $1^{\prime}$. Amplitude variation of model $1^{\prime}$ is smaller than that of the observation and dose not 
Table 3. Parameters used in the calculations.

\begin{tabular}{cccc}
\hline & Model & $A_{1}$ (barrier effect) & $A_{2}$ (source effect) \\
\hline model 1 & Source model & 1.0 & 2.0 \\
model 2 & CIR barrier model & $10.0 / 1.0$ & 0.0 \\
model 3 & Coupling model & $10.0 / 1.0$ & 2.0 \\
\hline
\end{tabular}

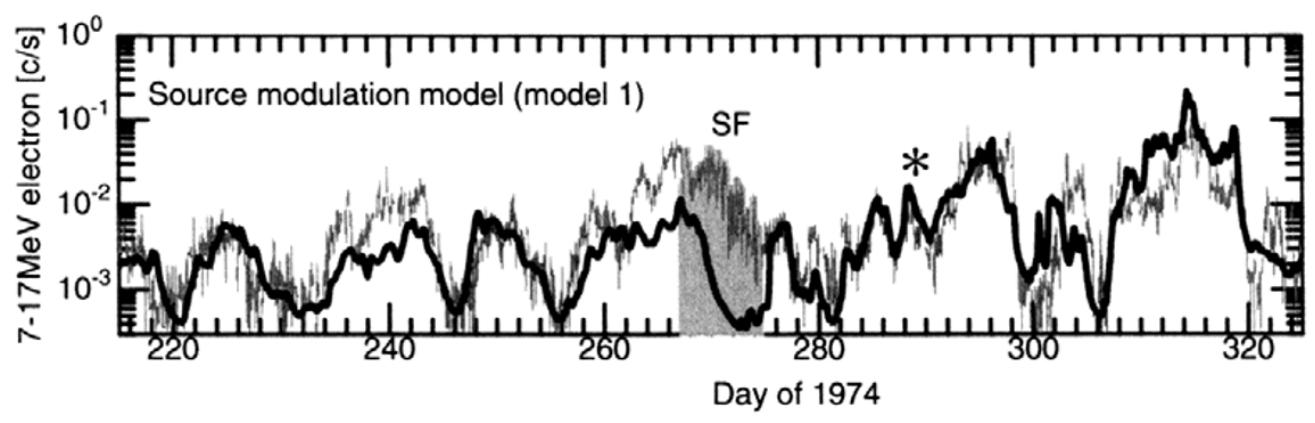

Fig. 7. The results of the calculation for model 1. The calculated electron variations at the position of Pioneer 11 (thick line) and observed 7-17 MeV electron variation (thin line) are shown. The calculated intensities are normalized by the value observed at day 285.5 (labeled "*”). A hatched portion of the observed data with label "SF" indicates the solar flare particle event.

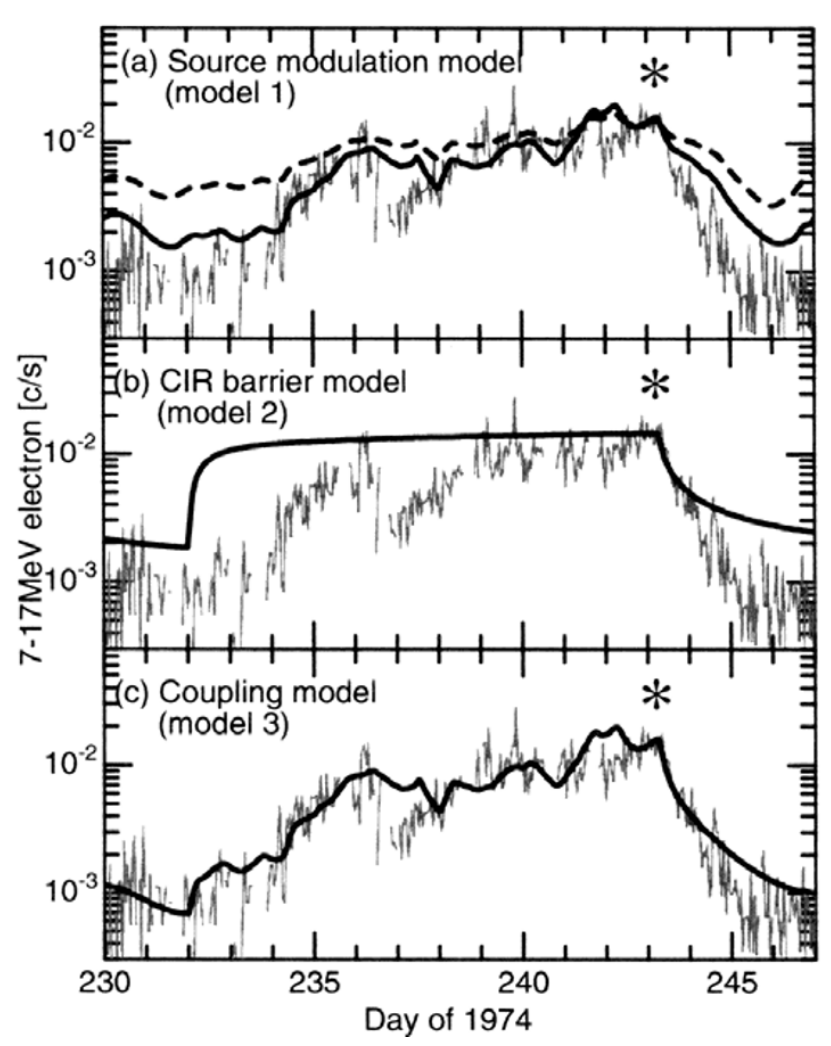

Fig. 8. (a) Variations of Jovian electrons calculated for model 1 (thick line; $A_{2}$ is 2.0 ) and model $1^{\prime}$ (dashed line; $A_{2}$ is 1.0 ), and the real observation (thin line) during the period of day 230-247, 1974. (b) Calculation for the barrier model (model 2, thick line) and observed electrons (thin line). (c) Calculation for the coupling model (model 3, thick line) and observed electrons (thin line). The calculated intensities are normalized by the value observed at day 243 (labeled “*”). agree with the actual variation well compared with model 1. Then, we confirm that the reasonable value of $A_{2}$ is 2.0 not 1.0 .

The thick line in panel (b) shows the result from model 2 (barrier effect by CIR). Although the flux decay at day 243 is reproduced well, this model does not explain the decrease of observed intensity during the period from day 232 to 235 . It is suggested that modulations of relativistic electrons with time scale of a few days are mainly caused by the source modulation at Jupiter.

Morioka and Tsuchiya (1996) concluded that the temporal variation of Jovian electrons observed in interplanetary space were explained by the coupling between source modulation at the Jovian magnetosphere and barrier effect caused by CIR. We examine the coupling using the present model (model 3). In panel (c), the coupling model shows coincidence with the observation better than the source model in panel (a). This result suggests that the coupling model explain the observed variations best.

We conclude that the release of Jovian electrons is modulated by the solar wind at Jupiter, and then the electron flux is affected by CIR during the transport in the interplanetary medium.

\section{Discussion}

\subsection{Source modulation by the solar wind}

The dynamic pressure has a predominant contribution to the electron modulations. We consider two different processes for the solar wind modulation;

1) The solar wind compression injects large amount of energy into the magnetosphere, and causes the acceleration of relativistic electrons and subsequent release of 
them into the interplanetary medium.

2) Variation in dynamic pressure of the solar wind changes the position of the Jovian magnetopause causing disturbances of magnetic and electric fields around the magnetopause, then the rate of electron diffusion (or transport) from the magnetosphere to interplanetary space is changed.

Between these two possibilities, we adopt the latter because the electron flux is decreased when the magnetosphere is compressed by the high-pressure solar wind. Furthermore, previous studies showed that the electron release process is independent of electron energy (Teegarden et al. (1974)). Therefore, as for the electron release processes, the possibility of (1) is considered to be low.

When we consider the cross-field diffusion as a mechanism of electron release, it is important to consider spatial and/or temporal scale of magnetic field fluctuations. If magnetic field fluctuation in space and time is comparable with the electron Larmor radius $R_{\mathrm{L}}$ or electron cyclotron frequency $\Omega_{\mathrm{e}}$ in the vicinity of the magnetopause of Jupiter, electrons can diffuse across the closed field line at the Jovian magnetopause. Typical magnitude of magnetic field magnitude $|B|$ is $\sim 5 \mathrm{nT}$ in the Jovian outer magnetosphere, $R_{\mathrm{L}}$ of $1 \mathrm{MeV}$ electron is an order of $\sim 10^{3} \mathrm{~km}$ and $\Omega_{\mathrm{e}}$ is an order of $10 \sim 100 \mathrm{~Hz}$. The outer magnetosphere is a very turbulent region which extends from $50 R_{\mathrm{J}}$ (Jovian radii) up to more than $100 R_{\mathrm{J}}$ responding to the dynamic pressure of the solar wind (Smith et al., 1978). When the magnetosphere expands due to the low solar wind pressure, the ratio of the turbulent component of the magnetic field $(\Delta B)$ to the background intensity $(B)$ at the magnetopause should increase rather than that in the case of the compressed magnetosphere. Thus the diffusion of energetic electrons across the magnetopause is expected to take place preferentially during the expanded magnetosphere.

According to our simulation, the observed electron flux is expressed by $f_{\mathrm{obs}} \sim P_{\mathrm{SW}}^{-2}$, which means significant influence of the solar wind to the release of Jovian electrons from the magnetosphere. Release rates of Jovian electrons were estimated by several authors. For example, Zhang et al. (1993) estimated $\sim 9 \times 10^{25}$ electrons $\cdot \mathrm{s}^{-1}$ for $5-10 \mathrm{MeV}$ electron from the observations of electron anisotropies in the magnetosheath. On the other hand, Mckibeen et al. (1993) estimated a total electron content for the outer magnetosphere of $1 \times 10^{27}$ electrons for $>16 \mathrm{MeV}$. Even if the differences in energy produces a difference of a factor of 10 in flux for an $E^{-3}$ spectrum (Mckibben et al., 1993), escape of the electrons into the interplanetary space become a significant loss of relativistic electrons in the outer magnetosphere. In order to supply a large number of relativistic electrons into the interplanetary medium, it is necessary to maintain strong acceleration and accumulation of energetic electrons in the Jovian outer magnetosphere during the period when high dynamic pressure of the solar wind interact with the magnetosphere. 5.2 Release efficiency controlled by the polarity of IMF

The polarity dependence of the electron release suggests magnetic reconnection between Jupiter's magnetic field and the IMF as one of the possible mechanism. Huddleston et al. (1997) and Walker and Russell (1985) showed evidences of flux transfer events (FTEs) from the Pioneers and Voyagers observations. Simpson et al. (1993) and Ferrando et al. (1993) also speculated the reconnection process to explain the burst-like increases of relativistic electron flux in interplanetary space. If the reconnection occurs, Jovian electrons escape easily into interplanetary space. In the case of the terrestrial magnetosphere, the reconnection process has an important roll on the magnetospheric phenomena, and has a dependence on the north-south polarity of the IMF. In our study, we found the away/toward polarity dependence for the Jovian electron fluxes. It is necessary to consider different reconnection regions and/or magnetic field configuration at the magnetopause region of Jupiter to explain the dawn/dusk asymmetry.

\subsection{Effect of CIR and diffusive transport in interplane- tary space}

The barrier effect of CIR on the modulation of Jovian electrons in interplanetary space was discussed by several authors (Conlon and Simpson, 1977; Conlon, 1978; Chenette, 1980). According to our research, variation of Jovian electron flux can not be explained only by the barrier effect although there is a contribution to decrease the observed Jovian electron fluxes.

\section{Conclusion}

We have investigated relationships between flux of Jovian high-energy electrons and variations of the solar wind in detail using the Pioneer 10, 11 and Ulysses data.

We conclude that energetic Jovian electron variations observed in interplanetary space are expressed as a function of the dynamic pressure of the solar wind and the polarity of IMF at the position of Jupiter, coupled with the transport effect by CIR. Our main results are summarized as follows.

1) From the cross-correlation analyses, we obtained clear evidence that the solar wind variation at Jupiter changes the electron release rates from the Jovian magnetosphere with an inverse relation, that is, the electron flux increases (decreases) when the dynamic pressure decrease (increase).

2) The polarity of the IMF at Jupiter controls the efficiency of electron release. In the dawn side region of the Jovian magnetosphere, intensity of Jovian electron flux is enhanced during the period when Jupiter is in the toward sector. On the other hand, in the dusk side of the Jovian magnetosphere, Jovian electron flux increases during the period when Jupiter is in the away sector.

3) A numerical calculation was made using a convective diffusion equation to evaluate the electron modulation processes in interplanetary space. The results of the calculation disclosed that mixture of source modulation and barrier of CIR could account for the observed flux variations in interplanetary space.

Acknowledgments. We express our gratitude to Prof. H. Oya for his helpful discussion. The Pioneer 10, 11 and Ulysses data were made available to us through the courtesy of the National Space Science Data Center. This work was supported by Japanese STEP 
Project, Ministry of Educations, Science and Culture, and Research Fellowships of the Japan Society for the Promotion of Science for Young Scientists.

\section{References}

Balogh, A., T. J. Beek, R. J. Forsyth, P. C. Hedgecock, R. J. Marquedant, E. J. Smith, D. J. Southwood, and B. T. Turutani, The magnetic field investigation on the Ulysses mission: Instrumentation and preliminary scientific results, Astron. Astrophys. Suppl. Ser, 92, 221, 1992.

Bame, S. J., D. J. McComes, B. L. Barraclough, J. L. Phillips, K. J. Sofaly, J. C. Chavez, B. E. Goldstein, and R. K. Sakurai, The Ulysses solar wind plasma experiment, Astron. Astrophys. Suppl. Ser., 92, 237-265, 1992.

Chenette, D. L., The propagation of Jovian electrons to Earth, J. Geophys. Res., 85(A5), 2243-2256, 1980.

Chenette, D. L., T. F. Conlon, and J. A. Simpson, Bursts of relativistic electrons from Jupiter observed in interplanetary space with the time variation of the planetary rotation period, J. Geophys. Res., 79, 35513558, 1974.

Chenette, D. L., T. F. Conlon, K. R. Pyle, and J. A. Simpson, Observations of Jovian electrons at $1 \mathrm{AU}$ throughout the 13 month Jovian synodic year, Astrophys. J., 215, L95-L99, 1977.

Conlon, T. F., The interplanetary modulation and transport of Jovian electrons, J. Geophys. Res., 83, 541-552, 1978.

Conlon, T. F. and J. A. Simpson, Modulation of Jovian electron intensity in interplanetary space by corotating interaction regions, Astrophys. J., 211, L45-L49, 1977.

Cooper, F. J., H. K. Mills, and J. H. King, Coordinated Heliospheric Observations $(\mathrm{COHO})$ data base, in Proceedings of the 22th International Cosmic Ray Conference, Dndlin, Vol. 3, pp. 796-799, 1991.

Ferrando, P., R. Ducros, C. Rastoin, A. Raviart, H. Kunow, R. Müller-Mellin, H. Sierks, and G. Wibberenz, Propagation of Jovian electrons in and out of the ecliptic, Adv. Space Res., 13, (6)107-(6)110, 1993a.

Ferrando, P., R. Ducros, C. Rastoin, and A. Raviart, Jovian electron jets in interplanetary space, Planet. Space Sci., 41, 839-849, 1993b.

Hamilton, D. C. and J. A. Simpson, Jovian electron propagation out of the solar equatorial plane: Pioneer 11 observations, Astrophys. J., 228 , L123-L127, 1979.

Huddleston, D. E., C. T. Rusell, G. Li, and A. Szabo, Magnetopause structure and the role of reconnection at the outer planets, J. Geophys. Res., 102 24289-2302, 1997.

McKibben, R. B., J. A. Simpson, and M. Zhang, Impulsive bursts of relativistic electrons discoverd during Ulysses's traversal of Jupiter's dusk-side magnetosphere, Planet. Space Sci., 41, 1041-1058, 1993.

Morioka, A. and F. Tsuchiya, Solar wind control of Jovian electron flux: Pioneer 11 analysis, Geophys. Res. Lett., 23, 2963-2966, 1996.

Morioka, A., F. Tsuchiya, and H. Misawa, Modulation of Jovian electrons by the solar wind, Adv. Space Res., 20, 205-208, 1997.
Pyle, K. R. and J. A. Simpson, The Jovian relativistic electron distribution in interplanetary space from 1 to $11 \mathrm{AU}$ : Evidence for a continuously emitting "point" source, Astrophys. J., 215, L89-L93, 1977.

Schardt, A. W., F. B. McDonald, and J. H. Trainor, Jovian modulation of interplanetary electrons as observed with Voyagers 1 and 2, J. Geophys. Res., 88(A3), 1989-2000, 1983.

Simpson, J. A., D. Hamilton, G. A. Lentz, R. B. McKibben, A. MogroCampero, M. Perkins, K. R. Pyle, A. J. Tuzzolino, and J. J. O’Gallagher, Protons and electrons in Jupiter's magnetic field: Results from the University of Chicago experiment on Pioneer 10, Science, 183, 306-309, 1974

Simpson, J. A., J. D. Anglin, A. Balogh, M. Bercovitch, J. M. Bouman, E. E. Budzinski, J. R. Burrows, R. Cravell, J. J. Connell, R. Ducros, P. Ferrando, J. Firth, M. Garcia-Munoz, J. Henrion, R. J. Hynds, B. Iwers, R. Jacquet, H. Kunow, G. Lentz, R. G. Marsden, R. B. McKibben, R Müller-Mellin, D. E. Page, M. Perkins, A. Raviart, T. R. Sanderson, H. Sierks, L. Treguer, A. J. Tuzzolino, K.-P. Wenzel, and G. Wibberenz, The Ulysses cosmic ray and solar particle investigation, Astron. Astrophys. Suppl. Ser., 92, 365-399, 1992.

Simpson, J. A., D. A. Smith, and M. Zhang, Jovian electron propagation in three dimensions of the heliosphere: The Ulysses investigations, $J$. Geophys. Res., 98(A12), 21129-21144, 1993.

Smith, E. J. and J. H. Wolfe, Observations of interaction regions and corotating shocks between one and five AU: Pioneer 10 and 11, Geophys. Res. Lett., 3(3), 137-140, 1976.

Smith, E. J., L. Davis, Jr., D. E. Jones, P. J. Coleman, Jr., D. S. Colburn, P. Dyal, C. P. Sonett, and A. M. A. Frandsen, The planetary magnetic field and magnetosphere of Jupiter: Pioneer 10, J. Geophys. Res., 79, 3501-3513, 1974.

Smith, E. J., R. W. Fillius, and J. H. Wolfe, Compression of Jupiter's Magnetosphere by the Solar Wind, J. Geophys. Res., 83(A10), 4733-4742, 1978.

Teegarden, B. J., F. B. McDonald, J. H. Trainor, W. R. Webber, and E. C. Roelof, Interplanetary $\mathrm{MeV}$ electrons of Jovian origin, J. Geophys. Res., 79, 3615-3622, 1974

Walker, R. J. and C. T. Russell, Flux transfer events at the Jovian magnetopause, J. Geophys. Res., 90, 7397-7404, 1985.

Wolfe, J. H., J. D. Mihalov, H. R. Collard, D. D. McKibben, L.A. Frank, and D. S. Intriligator, Pioneer 10 observations of the solar wind interaction with Jupiter, J. Geophys. Res., 79, 3489-3500, 1974

Zhang, M., J. A. Simpson, and R. B. McKibben, Relativistic electron flux anisotropies in the duskside of Jovian magnetosphere: a test for location and escape mechanism., Planet. Space Sci., 41, 1029-1040, 1993.

F. Tsuchiya (e-mail: tsuchiya@pparc.geophys.tohoku.ac.jp), A. Morioka, and H. Misawa 